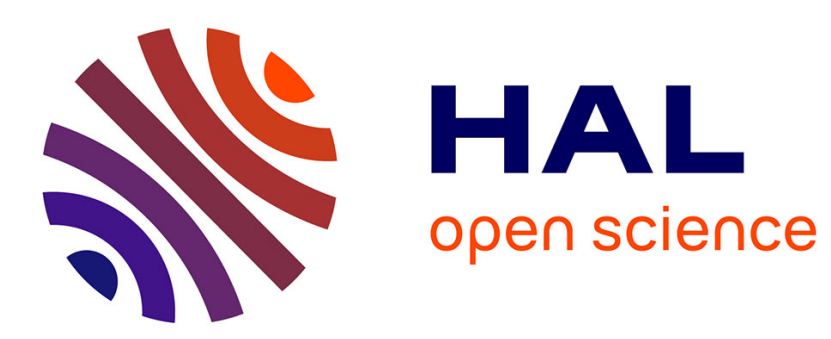

\title{
The rheological modelling of carbon nanotube suspensions in steady shear flows
}

Anson W. K. Ma, Francisco Chinesta, Malcolm R. Mackley, Amine Ammar

\section{To cite this version:}

Anson W. K. Ma, Francisco Chinesta, Malcolm R. Mackley, Amine Ammar. The rheological modelling of carbon nanotube suspensions in steady shear flows. International Journal of Material Forming, 2008, 1 (2), pp.83-88. 10.1007/s12289-008-0372-x . hal-01007374

\section{HAL Id: hal-01007374 https://hal.science/hal-01007374}

Submitted on 22 Jan 2017

HAL is a multi-disciplinary open access archive for the deposit and dissemination of scientific research documents, whether they are published or not. The documents may come from teaching and research institutions in France or abroad, or from public or private research centers.
L'archive ouverte pluridisciplinaire HAL, est destinée au dépôt et à la diffusion de documents scientifiques de niveau recherche, publiés ou non, émanant des établissements d'enseignement et de recherche français ou étrangers, des laboratoires publics ou privés.

\section{(c)(1)}

Distributed under a Creative Commons Attribution| 4.0 International License 


\title{
The rheological modelling of carbon nanotube (CNT) suspensions in steady shear flows
}

\author{
Anson W. K. Ma • Francisco Chinesta • \\ Malcolm R. Mackley • Amine Ammar \\ A. W. K. Ma $\cdot$ M. R. Mackley \\ Department of Chemical Engineering, University of Cambridge, \\ New Museums Site, Pembroke Street, \\ Cambridge CB2 3RA, UK \\ F. Chinesta $(\square)$ \\ Laboratoire de Mécanique des Systèmes et des Procédés, \\ 151 Boulevard de l'Hôpital, \\ 75013 Paris, France \\ e-mail: francisco.chinesta@paris.ensam.fr \\ A. Ammar \\ Laboratoire de rhéologie, Domaine universitaire, \\ 1301 Rue de la piscine, \\ 38041 Grenoble Cedex 9, France
}

\begin{abstract}
This paper is concerned with the rheological modelling of both chemically treated and untreated carbon nanotube (CNT) suspended in a Newtonian epoxy resin. CNT suspensions generally exhibited shear-thinning characteristic - the apparent viscosity decreases as shear rate increases - when subject to steady shear flows. Chemically treated CNT suspensions with little optical microstructure were found to exhibit a less significant shear-thinning effect compared with untreated CNT suspensions where clear optical aggregates were observed. In the case of treated CNT suspensions, the shear-thinning characteristic could be described using a Fokker-Planck based orientation model. The model assumed that the treated CNTs behaved as high aspect ratio rods and that shear flow was able to align the CNTs in the flow direction, thereby resulting in a decrease in the shear viscosity. Despite the success in describing the rheological response of treated CNT in steady shear flows, the orientation model failed to explain the more pronounced shear-thinning effect observed in untreated CNT suspensions having a hierarchy of aggregate structures. A new model called the aggregation/orientation (AO) model was formulated by modifying the Fokker-Planck equation. The AO model considered elements of aggregation as well as CNT orientation and it was capable of capturing the steady shear response of untreated CNT suspensions.
\end{abstract}

Keywords Fokker-Planck equation .

Aggregation/orientation (AO) model · Carbon nanotube

\section{Introduction}

The experimental rheology of carbon nanotube (CNT) suspensions has been studied by a number of authors (see for example [1-3]). In terms of steady shear measurements, addition of CNT to a Newtonian matrix has been found to increase the apparent viscosity at low shear and the viscosity decreases asymptotically to the matrix viscosity as shear rate increases [3-5]. This is known as steady shear-thinning and this type of behaviour is commonly observed in suspensions [6]. The extent of viscosity enhancement at low shear depends on a number of factors such as the state of dispersion as well as the type and aspect ratios of CNT used [7-9]. For untreated CNT suspensions where clear optical aggregates were present, an order of magnitude of increase in low shear viscosity could result by adding only $0.1 \%$ of CNTs $[3,4]$. In the case of chemically treated CNT suspensions, the viscosity enhancement effect tends to be less pronounced $[5,7,9]$. In this paper, the treated CNTs were supplied by Nanocomposites Inc. (USA) and they were chemically treated in a way such that aggregation was prevented by electrostatic repulsion between CNTs $[10,11]$. The untreated CNTs were produced by chemical vapour deposition (CVD) method [12] and were provided by the Department of Materials Science and Metallurgy, University of Cambridge.

This paper describes the modelling of steady shearthinning for both treated and untreated CNT suspensions. 
Detailed model formulation was reported in $[4,5]$ and more experimental data can be found in the article "The Microstructure and Rheology of Carbon Nanotube Suspensions" in this thematic issue of IJMF.

\section{Treated CNT suspensions-Fokker-Planck based orientation model}

In the modelling of steady shear-thinning response of treated CNTs, the CNTs were assumed to be an assembly of high-aspect-ratio rigid fibres subject to shear and rotary diffusion. Based on earlier work by Batchelor and coworkers [13-15], the constitutive equation for a dilute suspension with high-aspect-ratio rigid fibres can be written as:

$\sigma=-P \mathbf{I}+2 \eta \mathbf{D}+\boldsymbol{\tau}^{f}$

where $P$ denotes the pressure, $\mathbf{I}$ is the unit tensor, $\eta$ is the suspending medium viscosity, $\mathbf{D}$ is the strain rate tensor (symmetric component of the velocity gradient tensor). $\tau^{f}$ is the anisotropic viscous component of the stress tensor due to the presence of fibres, which is further given as:

$$
\left\{\begin{aligned}
\boldsymbol{\tau}^{f} & =2 \eta N_{p}(\mathbf{A}: \mathbf{D})+\beta D_{r}\left(\mathbf{a}-\frac{\mathbf{1}}{3}\right) \\
\mathbf{A} & =\int_{S(0,1)}(\boldsymbol{\rho} \otimes \mathbf{\rho} \otimes \mathbf{\rho} \otimes \mathbf{\rho}) \psi(\boldsymbol{\rho}) \mathrm{d} \boldsymbol{\rho} \\
\mathbf{a} & =\int_{S(0,1)}(\boldsymbol{\rho} \otimes \boldsymbol{\rho}) \psi(\boldsymbol{\rho}) \mathrm{d} \boldsymbol{\rho}
\end{aligned}\right.
$$

where $N_{p}$ is a scalar parameter that depends on the fibre concentration as well as the aspect ratio of fibres in dilute suspensions, $\mathbf{A}$ and a are the fourth-order and second order orientation tensors respectively, $\rho$ is the unit vector aligned in the fibre axis direction, $S(0,1)$ represents the surface of the unit sphere where the fiber orientation is defined, $\psi(\rho)$ is the fiber orientation distribution and $\beta$ is a parameter that depends on the number concentration of CNTs and temperature. $D_{r}$ is the rotary diffusion coefficient and physically it represents the rate of misalignment from the flow direction. Equation 2 was proposed for suspensions involving Brownian rods [16], with the second term on the right accounted for randomising Brownian motion. The second term is negligible in the case of steady shear and large deformation, but it is needed for describing the experimentally observed elasticity in Brownian rod systems. Larson considered only Brownian motion and the constitutive equation predicted Maxwell type elasticity when the suspension is subject to small-amplitude oscillatory viscoelastic measurements [16]. In general, it is however more appropriate to replace the Brownian diffusion term $\left(D_{r}\right)$ by an effective diffusion coefficient which accounts for all possible randomising events such as Brownian motion, tube-tube hydrodynamic interaction and electrostatic repulsion between CNTs $[5,17]$. A comprehensive review of short fibre suspension modelling is beyond the scope of this paper, but further information can be found in the review by [18] for example.

In terms of the mathematical treatment of CNT orientation distribution, closure approximations for $\mathbf{A}$ were avoided and the Fokker-Planck equation [Eq. 3] was used to ensure numerical accuracy (see for example [19]):

$\frac{\mathrm{d} \psi}{\mathrm{d} t}+\frac{\partial}{\partial \boldsymbol{\rho}}\left(\frac{\mathrm{d} \boldsymbol{\rho}}{\mathrm{d} t} \psi\right)=\frac{\partial}{\partial \boldsymbol{\rho}}\left(D_{r} \frac{\partial \psi}{\partial \boldsymbol{\rho}}\right)$

where the flow induced orientation term $\frac{d \rho}{d t}$ is usually modelled using the Jeffery's equation. The Fokker-Planck based orientation model has been applied to the treated CNT suspensions and Fig. 1 shows the model fitting of surface-treated CNT suspensions with three different CNT concentrations $(0.05 \%, 0.2 \%$ and $0.33 \%)$. The rotary diffusion coefficient was identified to be in the order of $0.005 \mathrm{~s}^{-1}$ based on the best fits to experimental data. It has been found that the use of a constant $D_{r}$ appeared to be sufficient in providing a reasonably good estimation of the steady shear viscosity for general engineering problems. In terms of low-shear-rate data, although the torque generated by $\dot{\gamma}_{R}<0.1 \mathrm{~s}^{-1}$ was too small to be measured accurately in experiments, the model predicted a low-shear-rate Newtonian plateau. The level of the plateau depends on the parameter $N_{p}$ which scaled linearly with the CNT concentration as shown in the inset figure of Fig. 1. The success of the model suggests that the CNTs are behaving essentially as high-aspect ratio rigid rods with a very low

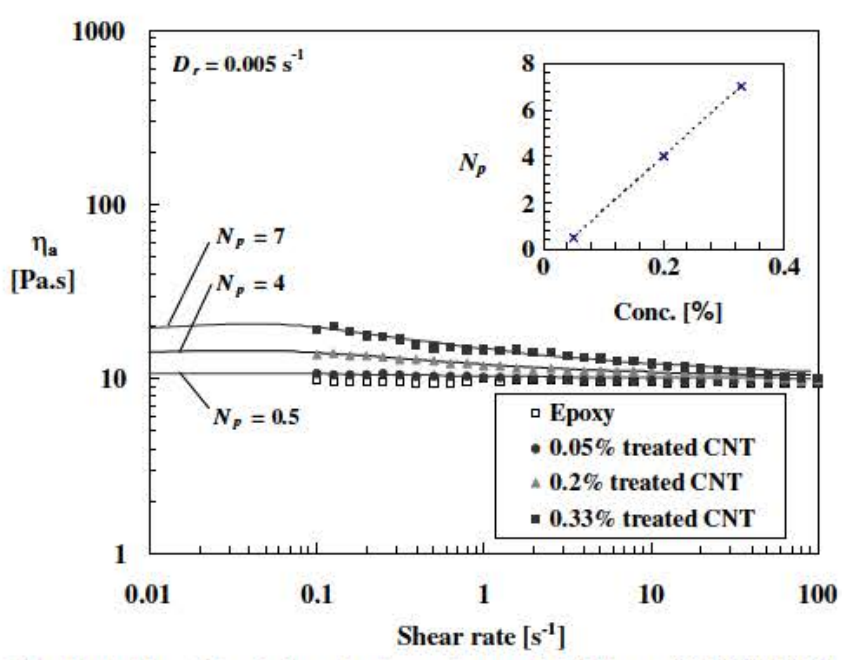

Fig. 1 Fokker-Planck based orientation model fitting of $0.05 \%, 0.2 \%$ and $0.33 \%$ treated CNT suspended in epoxy resin (Araldite LY556, Huntsman LLC). The model consists of two fittings parameters: $D_{r}$ and $N_{p}$. Best fits were obtained using $D_{r}=0.005 \mathrm{~s}^{-1}$ and the concentration-dependent parameter $N_{p}$ scaled linearly with the CNT concentration (inset figure) 
rotary diffusion coefficient. Experimental evidence of CNT alignment along the flow direction was reported by [3] and more recently, [9] showed cured CNT/epoxy samples where the CNTs were aligned by shear flow.

In terms of linear viscoelasticity (LVE), treated CNT suspensions showed milder elasticity compared with untreated CNT suspensions [7] and at present there is no universal consensus to describe experimental LVE data for surfactant-stabilised or treated CNT suspensions. [20] suggested that the experimentally observed elasticity was due to interaction between CNTs, whereas [7] proposed in their Maxwell-type modelling that elasticity was a result of stretching and bending of CNTs. Our recent work suggested that the mild elasticity observed in treated CNT suspensions could be described quantitatively using an appropriate rotary diffusion term to account for randomising events such as Brownian motion, tube-tube hydrodynamic interaction and electrostatic repulsion. The LVE modelling of treated CNT suspensions can be found in [5].

\section{Untreated CNT suspensions—aggregation/orientation model}

In the case of untreated CNT suspensions where clear optical aggregates were present, more significant steady shear thinning effect was generally observed [3]. In terms of optical microstructure, untreated CNT suspensions showed an optical texture which evolved depending on the shear conditions (Fig. 2). At low shear rates, coarsening of the optical texture was observed and at high shear rates, CNT suspensions were more uniformly dispersed and the aggregate size of CNTs decreased as shear rate increased.

It has been found that the Fokker-Planck based orientation model applicable to treated CNT suspensions could not describe the shear-thinning characteristic for untreated CNT suspensions. Figure 3 shows the fittings of experimental data using the orientation model with different fitting parameters $N_{p}$ and $D_{r}$. The level of the fitting curve is more sensitive to the absolute value of $N_{p}$ rather than $D_{r}$; however, it is also clear from all these fittings (Fig. 3a-d) that considering only CNT orientation was not sufficient in describing the observed viscosity enhancement and it is believed that the extra contribution to shear viscosity at low shear rates is due to the presence of CNT aggregates as observed in optical microstructure studies (Fig. 2).

A new model named the aggregation/orientation (AO) model was formulated. The model essentially considered a hierarchy of CNT aggregate structures in the untreated CNT suspension, where the shear viscosity was controlled not only by CNT orientation, but also the aggregation state of CNTs in the suspension. The Fokker-Planck description was modified to incorporate aggregation/disaggregation kinetics. The modified Fokker-Planck equation, which accounts for both CNT orientation and aggregation in a
Fig. 2 Optical microstructure of a $0.1 \%$ untreated CNT-epoxy suspension before $\left(0 \mathrm{~s}^{-1}\right)$ and after shearing at $0.5,10$ and $100 \mathrm{~s}^{-1}$ (shear time $=100 \mathrm{~s}$; optical depth $=130 \mu \mathrm{m}$; temperature $=25^{\circ} \mathrm{C}$ ). The untreated CNTs were supplied by the Department of Materials Science and Metallurgy at the University of Cambridge and the optical images were captured using the Cambridge Shear System (CSS 450; Linkam Scientific Instruments Ltd.)
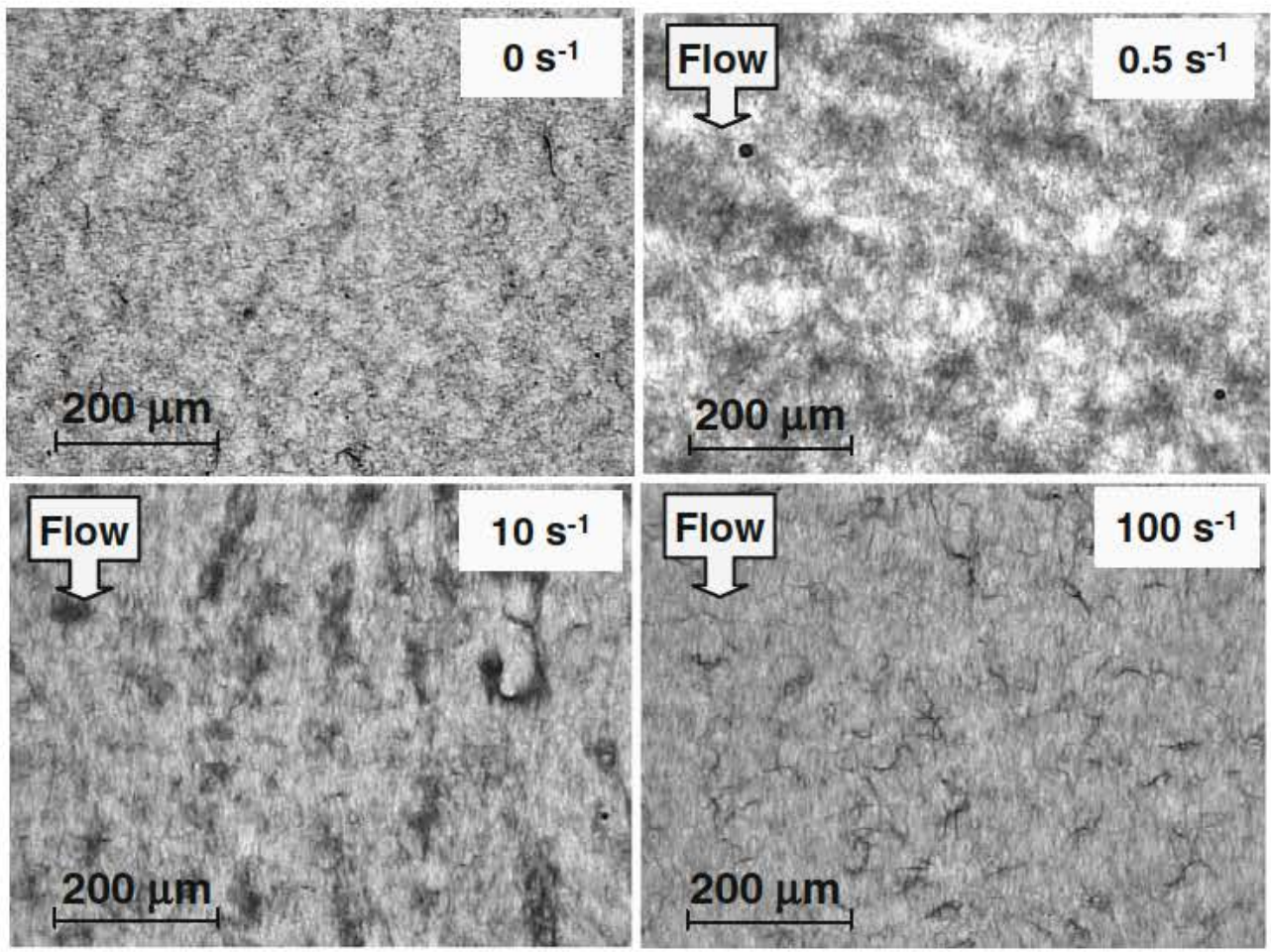
Fig. 3 Fokker Planck based orientation model fitting to experimental data of untreated CNT suspensions at two selected CNT concentrations (concentration $=0.25 \%$ for $\mathbf{a}$ and $\mathbf{c}$ and concentration $=0.1 \%$ for $\mathbf{b}$ and d). $\mathbf{a}$ and $\mathbf{b}$ showed the fittings with a fixed rotary diffusion coefficient $\left(D_{r}=0.0005 \mathrm{~s}^{-1}\right)$ and a changing $N_{p}$, whereas $\mathbf{c}$ and $\mathbf{d}$ showed the fittings with a fixed $N_{p}$, but with an adjustable $D_{r}$ Experimental data are represented by unfilled circles and the lines are the orientation model fits [4]
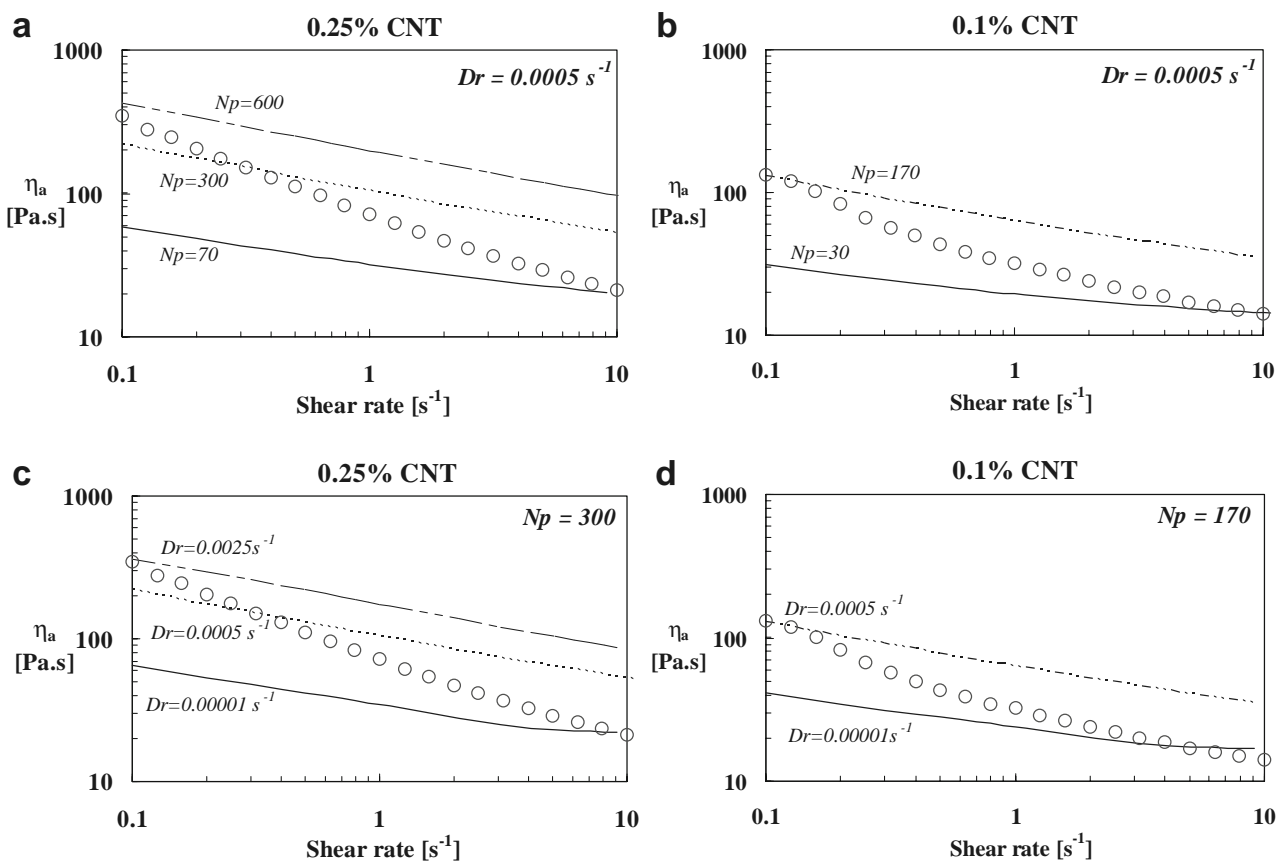

homogenous flow at steady state, is shown below. Detailed

derivation can be found in [4].

$$
\begin{aligned}
& \left\{-\frac{\partial}{\partial \boldsymbol{\rho}}\left\{\psi(\boldsymbol{\rho}, n) \frac{d \boldsymbol{\rho}}{d t}\right\}+\frac{\partial}{\partial \boldsymbol{\rho}}\left\{D_{r}(n) \frac{\partial \psi(\boldsymbol{\rho}, n)}{\partial \boldsymbol{\rho}}\right\}\right\}+ \\
& \quad+\left\{v_{c} \int_{r=0}^{r=n} \psi(\boldsymbol{\rho}, r) d r+v_{d} \int_{r=n}^{r=1} \psi(\boldsymbol{\rho}, r) d r-v_{d} \psi(\boldsymbol{\rho}, n) \int_{r=0}^{r=n} d r-v_{c} \psi(\boldsymbol{\rho}, n) \int_{r=n}^{r=1} d r\right\}=0
\end{aligned}
$$

Fig. 4 Aggregation/orientation (AO) model fitting to experimental data of untreated CNT suspensions at different CNT concentrations: a $0.25 \%$, b $0.1 \%$, c $0.05 \%$ and d $0.025 \%$. Experimental data are represented by unfilled circles. The values of $D_{r}^{\max }$ and $\beta$ were fixed for all the reported concentrations (where $D_{r}^{\max }=0.001 \mathrm{~s}^{-1}$ and $\beta=0.004$ ) and $N_{p}^{\max }$ was the only adjustable parameter in matching the experimental data with the model

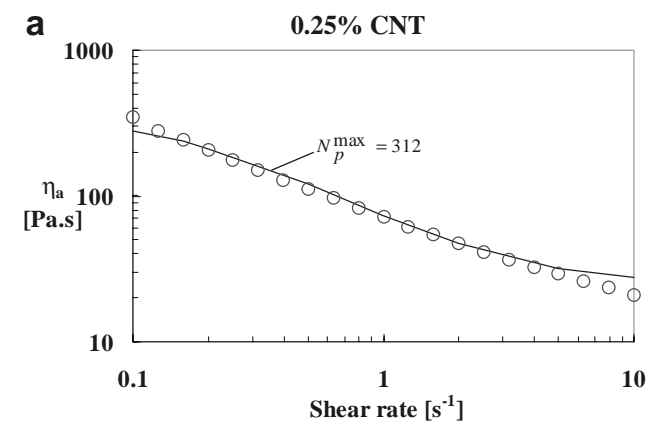

b
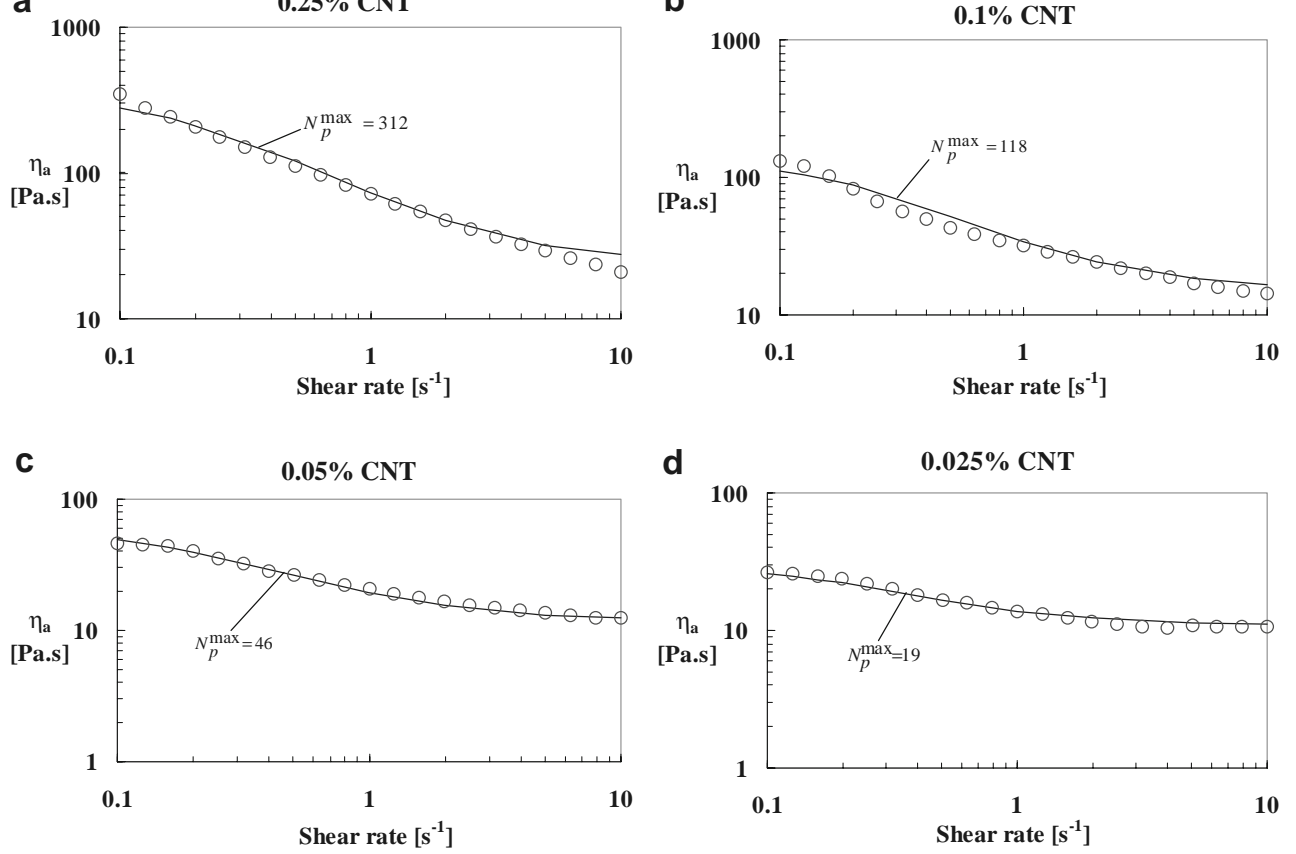

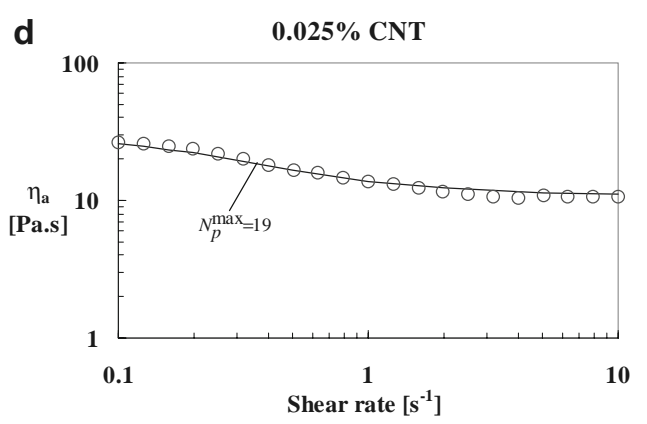


where $n \in[0,1]$ describes the state of aggregation $(n=0$ corresponds to CNTs that are free from entanglement and $n=1$ represents a CNT aggregate network); $v_{c}$ is the aggregation velocity and $v_{d}$ is the disaggregation velocity. The constitutive equation becomes:

$\boldsymbol{\tau}^{f}=2 \int_{0}^{1} \eta N_{p}(n)(\mathbf{A}(n): \mathbf{D}) \mathrm{d} n$

where the contribution of the rotary diffusion has been neglected and

$\mathbf{A}=\int_{S(0,1)}(\boldsymbol{\rho} \otimes \boldsymbol{\rho} \otimes \boldsymbol{\rho} \otimes \boldsymbol{\rho}) \psi(\boldsymbol{\rho}, n) \mathrm{d} \boldsymbol{\rho}$

To simplify the analysis and minimize the number of rheological parameters, the following assumptions are made:

1. $N_{p}$ varies linearly with the population variable $n$. Moreover, as $N_{p}^{\min }=N_{p}(n=0) \ll N_{p}^{\max }=N_{p}(n=1)$ we can assume $N_{p}^{\min } \approx 0$ leading to Eq. 7a where $N_{p}(n) \approx N_{p}^{\max } n$.

2. $D_{r}$ is mainly due to CNT interactions and it vanishes in the limit case of $n=1$ where the only orientation mechanism is affine deformation, as typically assumed in the case of associative polymers [21, 22]. A linear dependence on the parameter $n$ is assumed as described by Eq. $7 \mathrm{~b}$.

3. $v_{d}$ increases linearly with the shear rate and it takes the form as given in Eq. 7c where $\dot{\gamma}_{\max }$ is a characteristic shear rate above which the suspension viscosity coincides with the matrix viscosity. At high shear rates, $v_{d}$ remains constant and $v_{d}=v_{d}^{\max }$ (Eq. 7d)

4. $v_{c}$ decreases linearly with the shear rate and it takes the form as given in Eq. 7e. It can be noted from Eq. $7 \mathrm{f}$ that as the shear rate becomes higher than $\dot{\gamma}_{\max }$, the aggregation velocity becomes zero.

$$
\left\{\begin{array}{c}
N_{p}(n)=N_{p}^{\max } n+N_{p}^{\min }(1-n) \approx N_{p}^{\max } n \\
D_{r}(n)=D_{r}^{\max }(1-n) \\
v_{d}(\dot{\gamma})=\left\{\begin{array}{cl}
v_{d}^{\max }\left(\frac{\dot{\gamma}}{\dot{\gamma}_{\max }}\right) & \text { if } \dot{\gamma} \leq \dot{\gamma}_{\max }(\mathrm{c}) \\
v_{d}^{\max } & \text { if } \dot{\gamma}>\dot{\gamma}_{\max }(\mathrm{d})
\end{array}\right. \\
v_{c}(\dot{\gamma})=\left\{\begin{array}{cl}
v_{c}^{\max }\left(\frac{\dot{\gamma}_{\max }-\dot{\gamma}}{\dot{\gamma}_{\max }}\right) & \text { if } \dot{\gamma} \leq \dot{\gamma}_{\max }(\mathrm{e}) \\
0 & \text { if } \dot{\gamma}>\dot{\gamma}_{\max }(\mathrm{f})
\end{array}\right.
\end{array}\right.
$$

The rheological model contains three independent fitting parameters: the concentration and aspect-ratio parameter $\left(N_{p}^{\max }\right)$, the rotational diffusion coefficient $\left(D_{r}^{\max }\right)$ and the ratio between the aggregation and disaggregation velocities $\left(\beta=v_{c} / v_{d}\right)$. Figure 4 shows the best fits to experimental data with different CNT concentrations. In these fittings, it was assumed that only $N_{p}^{\max }$ depended on the CNT concentration and the other two rheological parameters were not a function of the concentration (i.e. $D_{r}^{\max } \neq f(\phi)$ and $\beta \neq f(\phi))$. As shown in Fig. 4, reasonable agreement between experimental results and the AO model was obtained with $D_{r}^{\max }=0.001 \mathrm{~s}^{-1}$ and $\beta=0.004$, further supporting the belief that the more pronounced shear-thinning in untreated CNT suspensions was due to the orientation as well as the aggregation of CNTs.

\section{Conclusions}

The steady shear-thinning characteristics of both chemically treated and untreated carbon nanotube (CNT) suspensions have been modelled. Experimentally, untreated CNT suspensions were found to exhibit a more pronounced shear-thinning effect compared with treated CNT suspensions. The comparatively moderate shear-thinning behaviour of treated CNT suspensions was successfully modelled using a Fokker-Planck based orientation model with two fitting parameters $\left(N_{p}\right.$ and $\left.D_{r}\right)$. The model essentially assumed treated CNTs as short and rigid fibres that can align in shear flow and shear thinning was believed to be a result of progressive alignment of CNTs in the flow direction.

In the case of untreated CNT suspensions, the FokkerPlanck orientation model failed to describe the experimental shear thinning. In-situ optical observations clearly showed the presence of a hierarchy of CNT aggregate structures which evolved as the magnitude of shear rate changed. A new model named aggregation/orientation (AO) model was formulated. The model considered the effect of CNT orientation and CNT aggregation and the Fokker-Planck equation was modified accordingly. An additional fitting parameter representing the kinetics of CNT aggregation/disaggregation was introduced in the modelling and the resulting $\mathrm{AO}$ model with three fitting parameters was capable of describing experimental data of untreated CNT suspensions. The model was supported by experimental optical observations and offered a plausible explanation to the significant shear-thinning characteristic of aggregating untreated CNT suspensions.

\section{References}

1. Kinloch IA, Roberts SA, Windle AH (2002) A rheological study of concentrated aqueous nanotube dispersions. Polymer 43:74837491

2. McNally T, Pöschke P, Halley P, Murphy M, Martin D, Bell SEJ, Brennan GP, Bein D, Lemoine P, Quinn JP (2005) Polyethylene multiwalled carbon nanotube composites. Polymer 46:8222-8232

3. Rahatekar SS, Koziol KKK, Butler SA, Elliott JA, Shaffer MSP, Mackley MR, Windle AH (2006) Optical microstructure and 
viscosity enhancement for an epoxy resin matrix containing multiwall Carbon Nanotubes. J Rheol 50:599-610

4. Ma AWK, Chinesta F, Ammar A, Mackley MR (2008a) Rheological modelling of Carbon Nanotube aggregate suspensions. J Rheol (in press)

5. Ma AWK, Chinesta F, Mackley MR (2008b) The rheology and modelling of chemically treated Carbon Nanotube suspensions. J Rheol (in press)

6. Cross MM (1965) Rheology of non-Newtonian fluids: a new flow equation for pseudo-plastic systems. J Colloid Interface Sci 20:417-437

7. Xu J, Chatterjee S, Koelling KW, Wang Y, Bechtel SE (2005) Shear and extensional rheology of carbon nanofiber suspensions. Rheol Acta 44:537-562

8. Huang YY, Ahir SV, Terentjev EM (2006) Dispersion rheology of carbon nanotubes in a polymer matrix. Phys Rev B 73:125422-1-9

9. Fan Z, Advani SG (2007) Rheology of multiwall carbon nanotube suspensions. J Rheol 51:585-604

10. Dyke CA, Tour JM (2003) Unbundled and highly functionalized carbon nanotubes from aqueous reactions. Nano Lett 3:1215-1218

11. Dyke CA, Tour JM (2004) Overcoming the insolubility of carbon nanotubes through high degrees of sidewall functionalization. Chem Eur J 10:812-817

12. Singh C, Shaffer MSP, Windle AH (2003) Production of controlled architectures of aligned Carbon Nanotubes by an injection chemical vapour deposition method. Carbon 41:359-368
13. Batchelor GK (1970) The stress system in a suspension of forcefree particles. J Fluid Mech 41:545-570

14. Hinch EJ, Leal LG (1975) Constitutive equations in suspension mechanics. Part I. J Fluid Mech 71:481-495

15. Hinch EJ, Leal LG (1976) Constitutive equations in suspension mechanics. Part II. J Fluid Mech 76:187-208

16. Larson RG (1999) The structure and rheology of complex fluids. Oxford University Press, New York, pp 261-355

17. Folgar F, Tucker CL (1984) Orientation behaviour of fibers in concentrated suspensions. J Reinf Plast Compos 3:98-119

18. Petrie CJS (1999) The rheology of fibre suspensions. J NonNewtonian Fluid Mech 87:369-402

19. Hinch EJ, Leal LG (1972) The effect of Brownian motion on the rheological properties of a suspension of non-spherical particles. J Fluid Mech 52:683-712

20. Hough LA, Islam MF, Janmey PA, Yodh AG (2004) Viscoelasticity of single wall Carbon Nanotube suspensions. Phys Rev Lett 93:168102-1-4

21. Vaccaro A, Marrucci G (2000) A model for the nonlinear rheology of associating polymers. J Non-Newtonian Fluid Mech 92:261273

22. Hernandez Cifre JG, Barenbrug TkhMAOM, Schieber JD, van den Brule BHAA (2003) Brownian dynamics simulation of reversible polymer networks under shear using a noninteracting dumbbell model. J Non-Newtonian Fluid Mech 113:73-96 